Hope College

Hope College Digital Commons

Faculty Publications

$2-2013$

\title{
Attachment and 'Hikikomori': A Psychosocial Developmental Model
}

Alexander Krieg

Hope College

Jane R. Dickie

Hope College, dickie@hope.edu

Follow this and additional works at: https://digitalcommons.hope.edu/faculty_publications

Part of the Psychology Commons

\section{Recommended Citation}

Krieg, Alexander and Jane R. Dickie. "Attachment and 'Hikikomori': A Psychosocial Developmental Model." International Journal of Social Psychiatry 59, no. 1 (2013) http://dx.doi.org/10.1177/0020764011423182

This Article is brought to you for free and open access by Hope College Digital Commons. It has been accepted for inclusion in Faculty Publications by an authorized administrator of Hope College Digital Commons. For more information, please contact digitalcommons@hope.edu. 


\section{Attachment and hikikomori: A psychosocial developmental model}

\author{
Alexander Krieg' and Jane R. Dickie'
}

International Journal of

Social Psychiatry

$1-12$

(C) The Author(s) 2011

Reprints and permissions: sagepub.co.uk/journalsPermissions.nav DOI: |0.1|77/00207640||423|82 isp.sagepub.com

(S)AGE

\begin{abstract}
Background: Hikikomori (acute social withdrawal) is a social issue in Japan that affects both the society and the lives of the individual sufferers.

Aims: This study aims to connect attachment theory and hikikomori by using a culturally sensitive psychosocial developmental model that outlines the various stages of attachment throughout the developmental years.

Method: Twenty-four hikikomori sufferers and 60 comparison group participants were given questionnaires assessing parent and peer relationships, temperament and school experiences.

Results: We found the hikikomori participants had a higher incidence of ambivalent attachment, reported more parental and peer rejection and bullying, and expressed greater temperamental shyness. Path analysis supported our developmental model. We found that shy temperament and parental rejection predicted ambivalent attachment, which when coupled with peer rejection predicted hikikomori. Our model implies that treatment and prevention may require attention to attachment insecurities in early childhood, peer rejection in middle childhood and/or early adolescence.

Conclusion: We believe it is helpful in understanding hikikomori to first understand how the attachment system balances security with exploration and the anxiety associated with novelty and challenge. Finally, we examine implications of the model for intervention, treatment and future research.
\end{abstract}

\title{
Keywords
}

hikikomori, social withdrawal, attachment, peer rejection, Japan

\section{Introduction}

The term hikikomori, from the Japanese word meaning 'to pull away', describes a condition of acute social withdrawal, where individuals shut themselves away from society for months or years. Hikikomori is used to describe both the condition of withdrawal and the sufferer. While this term was coined by Saito (2003) in Japan, this form of acute social withdrawal has been observed all over the world in countries such as Hong Kong (Wong, 2009), Oman (Sakamoto, Martin, Kumano, Kuboki, \& Al Adawi, 2005), Spain (Garcia-Campayo, Alda, Sobradiel, \& Sanz, 2007), Australia and the UK (Wong, 2009). This report, however, focuses on hikikomori in Japan, where it has received media attention in recent years (Asahi Shinbun, 2000; Larimer, 2001; Rees, 2002) and where there are documented increases in adolescent and young adult social withdrawal (Furlong, 2008; Zielenziger, 2006). The best estimates of hikikomori sufferers range from 410,000 in a survey conducted by the University of Okinawa in 2002
(Miyake, 2002) to 1.5 million estimated at risk by the Japanese Ministry of Health, Labour and Welfare (Aoki, 2010). Hikikomori represents a terrible loss on both a personal and societal level and compels further research to specify etiology and factors that contribute towards ameliorating this problem.

Hampering the search for the prevention and treatment of hikikomori is the difficulty in defining it (Teo \& Gaw, 2010). Is it a separate disorder culturally specific to Japan as Saito (1998, 2003) and Hattori (2005) have suggested, or is it a symptom of comorbid psychological disorders such as major depressive disorder, social phobia, agoraphobia or some

'Department of Psychology, Hope College, Holland, Michigan, USA

\section{Corresponding author:}

Jane R. Dickie, Professor of Psychology, Hope College, Department of Psychology 35 E I2th Street, Holland, MI 49422-9000, USA

Email: dickie@hope.edu 
personality disorders (American Psychiatric Association, 2000; Teo \& Gaw, 2010)? While some studies (Borovoy, 2008; Koyama, Miyake, Kawakami, Tsuchiya, \& Tachimori, 2010; Nakajima, Tsukamoto, Ooshige, Kishi, \& Oota, 2008; Suwa \& Suzuki, 2002; Tsujimoto, Daimon, Izumi, Sawai, \& Iwashige, 2007) reported that the majority of hikikomori who sought treatment were classified by other existing psychiatric disorders, the government estimate of the comorbidity surrounding hikikomori was only 35\% (Ministry of Health, Labour and Welfare, 2003). (See Teo (2010) for a wellorganized overview of hikikomori.) For the purposes of this paper, we define hikikomori as a behaviour containing both elements of social withdrawal (non-participation in society for at least six months) and social isolation (the discontinuation of relationships outside of the family during the time of withdrawal).

In this paper we propose a psychosocial developmental model to understand hikikomori. Previous work has cited parental relationships or peer rejection as possible factors in the etiology of hikikomori, but has failed to integrate the developmental importance of attachment in infancy and its relationship to peer rejection in childhood and adolescence. Previous studies have also lacked an independent measure of attachment. This model suggests that attachment, moulded by maternal behaviour shaped by culture, as well as dispositional variables, in addition to peer rejection may combine as risk factors leading to hikikomori. We finish by describing the implications of the model for intervention, treatment and future research.

\section{Overview of attachment}

The psychosocial developmental path for hikikomori proposed in this report relies heavily on the theory of attachment. Granqvist and Dickie (2006) previously outlined the description of this affectional bond between offspring and their caregivers. Bowlby $(1973,1982)$ proposed that infants possess an attachment behavioural system, which is initiated when infants signal behaviours (e.g. crying, screaming, smiling, following) during situations that they consider threatening. If the caregivers are responsive, the predictable outcome of these signalling behaviours is physical proximity between the offspring and the caregiver. Secure attachment system functioning is evident when infants turn to their attachment figures when distressed (i.e. safe haven behaviours) or when exploring their environment (i.e. secure base behaviours), which enables them to attain confidence for further exploration. Bowlby also argued that the way the caregiver responds in attachment-activating situations creates the child's internal working models (i.e. cognitive-affective representations of self and others). Internal working models are then responsible for the continuity in attachment functioning.

Attachment theory has been helpful in understanding children's socioemotional development (see Cassidy \&
Shaver, 1999). For example, attachment security in infancy predicts empathy, social competence and ego resilience, while attachment insecurity predicts externalizing (e.g. aggression, conduct problems) as well as internalizing (e.g. social anxiety, psychosomatic complaints) behaviour problems throughout childhood and adolescence (e.g. Weinfield, Sroufe, Egeland, \& Carlson, 1999).

The important point in this context is that securely attached children function well because they balance proximity seeking and exploration. Caregiver sensitivity in handling the child's distress ultimately gives the child confidence to explore new situations as well as demonstrates that others can be trusted to help and not harm the child in times of need or distress. The securely attached child avoids being overwhelmed by fear and dread (Cassidy, 1994). In contrast, the insecurely attached child either explores defensively avoiding contact with a rejecting caregiver (avoidant attachment) or clings passively to an inconsistent caregiver at the expense of exploration (ambivalent attachment). In the most extreme case, stress provokes a breakdown in organized behaviour (disorganized attachment) in the face of a frightened or frightening caregiver (Ainsworth, Blehar, Waters, \& Wall, 1978; Main \& Hesse, 1990).

In the last 20 years, scholars have investigated attachment beyond childhood to understand how the attachment functions of feeling safe enough to explore novelty and new relationships are transferred from primary caregivers to others. As young children move further from the proximal protection of caregivers and spend more time with peers, they begin to transfer the safe haven functions of their caregivers to relationships with peers. In early adulthood, reciprocal relations with partners serve as a secure base, replacing relations with peers and parents, creating feelings of relative safety in the adult relationship that permit risk-taking (Fraley \& Davis, 1997; Friedlmeier \& Granqvist, 2006; Hazan \& Zeifman, 1999).

\section{Factors related to early infant attachment and their measurements}

As mentioned in our overview of attachment, maternal sensitivity is essential to the development of secure attachment (Bowlby, 1969), and much empirical evidence supports this claim (Cunha, Soares, Pinto-Gouveia, 2008; Goldsmith \& Alansky, 1987; Isabella, 1993). Additionally, parental behaviour associated with anxious and ambivalent attachment in children includes parents being overprotective and controlling (Vertue, 2003) as well as threatening and rejecting (Genuis, 1994; Scher, 2000). Other parental factors that affect anxious attachment are regular non-parental care, parental neglect, lack of physical proximity, threats of abandonment or harm, family instability and sexual abuse (Genuis, 1994). In Hattori's 2005 study, the majority of hikikomori clients reported negative and rejecting experiences with their parents although no direct measures of 


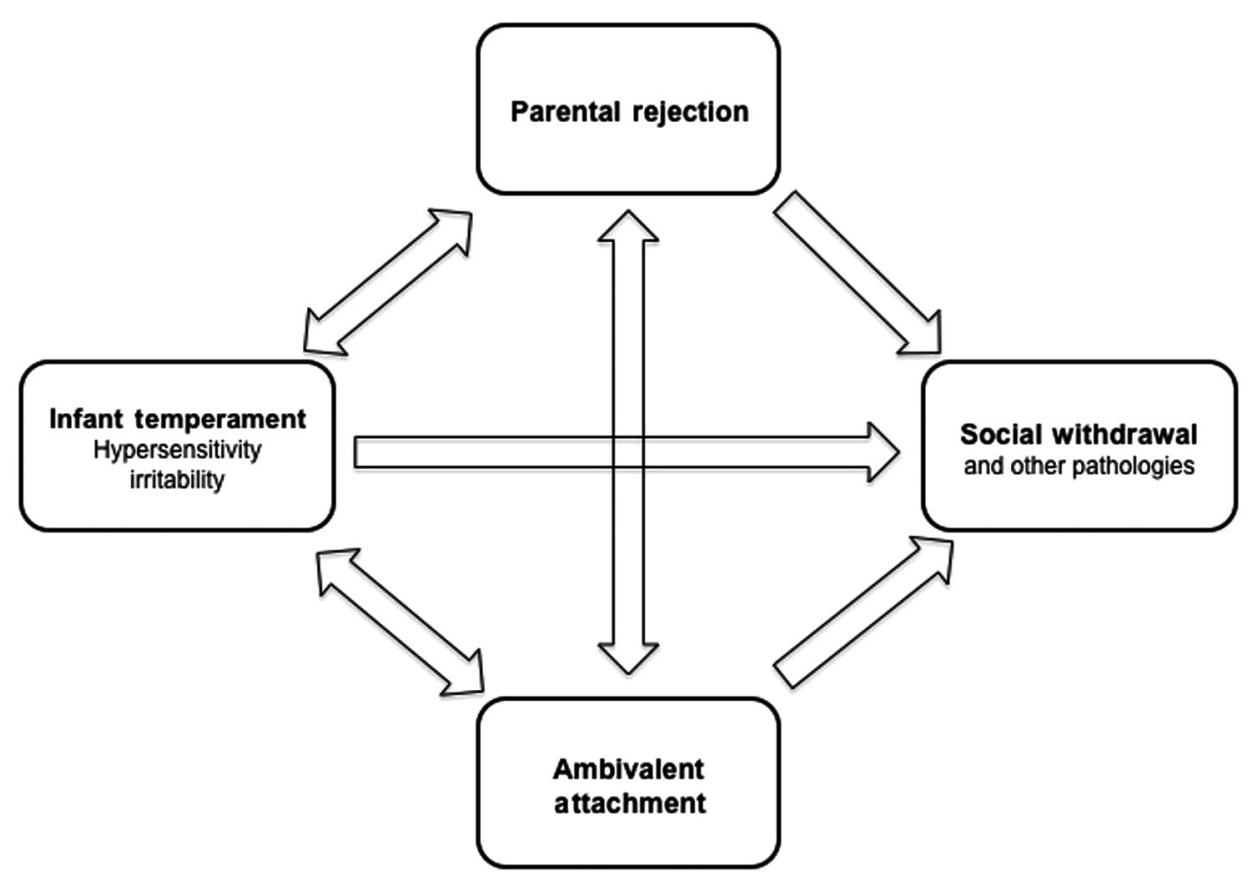

Figure I. Interconnections between infant temperament, parental behaviour, attachment and social withdrawal.

attachment were used. This is further supported by Koshiba (2007), who compared several aspects of family functioning between a hikikomori sample, an autistic sample and a control group. On problem solving, communication, affective responsiveness and overall general functioning, hikikomori families were significantly lower than that of both the control group and the families with autistic children.

Despite all of the evidence of the impact of parental behaviour on attachment, this is not the only process that affects attachment. Attachment style also depends on the dispositional characteristics of the child. It is apparent that the temperament of the child influences parental behaviour (Plomin \& Bergeman, 1991). For instance, if a child is slow to warm up or has a generally irritable temperament, caregivers would find it more difficult to regularly reciprocate warmth and sensitivity (Maccoby \& Jacklin, 1987; Thomas $\&$ Chess, 1977). Because of this, it is common to see children who are irritable in the first three to four months classified as ambivalently attached at 12 months (Chen \& Miyake, 1986). An individual's temperament may be predominantly a genetic characteristic (Grossman, Grossman, \& Waters, 2005) and shows remarkable stability in some dimensions (Miyake, Campos, Kagan, \& Bradshaw, 1986).

Like temperament, there may be other predispositions that affect attachment style and social anxiety. Aron (1999) studied what he called 'Highly Sensitive Persons', which he described as those who were born with a dispositional trait that makes them sensitive to subtle stimuli, easily over-stimulated and unsettled by novelty. According to his studies, this characteristic makes a child more vulnerable to inadequate or inconsistent caregiving (Aron, 1999). Figure 1 illustrates the interconnections between infant temperament, or disposition, attachment, parental rejection and social withdrawal.

Parent-child attachment has been studied across cultures, and there have been some differing interpretations of Japanese children's insecure attachment (Lamb, Thompson, Gardner, \& Charnov, 1985; Takahashi, 1990; Ujiie \& Miyake 1984; Van Ijzendoorn \& Kroonenberg, 1988). Attachment distribution among infants in Japan may differ from that in the USA. In US samples the distribution is 22\% Insecure/Avoidant, $65 \%$ Secure and 13\% Insecure/ Ambivalent (Ainsworth et al., 1978), whereas in Japan, Miyake, Chen, and Campos (1985) reported 0\% Insecure/ Avoidant, $75 \%$ Secure and $21 \%$ Insecure/Ambivalent, along with a few that could not being classified. However, even if there are different cultural responses to the infant measures of attachment, indications of secure attachment and insecure attachment still have meaning and long-term implications for Japanese infants (Rothbaum, Pott, Azuma, Miyake, \& Weisz, 2000; Rothbaum, Weisz, Pott, Miyake, \& Morelli, 2000; Yukawa, Tokuda \& Sato, 2007) Yukawa, Tokuda, \& Sato, 2007) and particular importance for social withdrawal.

\section{Attachment and social withdrawal}

In a longitudinal study of 1,092 American children, BoothLaForce and Oxford (2008) found that the combination of early insensitive parenting, insecure attachment and dysregulated temperament contributed to social withdrawal in elementary school children aged six to 12 . While it may 
superficially seem that the act of withdrawing from society would have a greater correlation with avoidant attachment, in a review on attachment and social withdrawal, Hastings, Nuselovici, Rubin, and Cheah (2010) found that ambivalently attached infants have difficulty coping with new challenges or social situations, and because of their fear of failure and rejection, they withdraw from social interactions. While the impact of parenting behaviours and attachment on shyness and social withdrawal has been studied primarily in infancy, there have also been a few studies that focus on middle childhood, adolescence and adulthood (for a review, see Hastings et al. 2010). In adolescence, a longitudinal study by Rubin, Chen, McDougall and Bowker (1995) reported that socially withdrawn 11 -year-olds, felt insecure and disconnected from their parents at age 14 .

Likewise, in Japan, some leading experts suggest that disruption in the parent-child relationship correlates with hikikomori (Furlong, 2008; Kawanishi, 2006; Teo, 2010), which is supported by clinical observations and case studies (Hattori, 2005; Sakamoto et al., 2005). Some specific rejecting behaviours claimed to upset this attachment relationship were ignoring the child (a practice called mushi), threatening to cut off the relationship with the child and locking the child out of the house - simulating actual abandonment (Hattori, 2003; 2005). These behaviours are also noted in Lebra's (1976) ethnography of Japanese parenting.

In addition to rejecting behaviours, Rubin, Hastings, Stewart, Henderson, and Chen (1997) linked overprotective, over-solicitous parenting, including intrusive micromanagement of the child's activities with overly strong affection outside of distress or need of comforting, to withdrawn behaviours. Japanese mothers have been reported to have a tendency to focus their children's attention on their relationship, representing themselves as the source of comfort, nurturance and protection (Rothbaum, Pott, et al., 2000; Rothbaum, Weisz, et al., 2000; Vogel \& Vogel, 1961), while minimizing any stress that the child experiences (Caudill \& Weinstein, 1969). This may be because mothers have primary responsibility for their infants' actions, especially those deemed negative. In order to avoid criticism from husbands and neighbours, mothers strive to keep their children from fussing, crying or maintaining a negative affect (Miyake et al., 1985). Often, because of the impact this has on the child's psyche, Japanese mothers are often lifelong sources of attachment (Lebra, 1976). It seems as if emotionally manipulative over-control, whether extremely affectionate or cold and negative, may put children on trajectories toward shyness and social withdrawal (Hastings et al., 2010), especially through the impact it has on fostering ambivalent attachment between mothers and children.

\section{Attachment, culture and peer relationships}

In school, children may be challenged in developing their first peer groups. Children's attachment styles tend to be stable in childhood and predict peer relationships (Bowlby, 1969; Grossman, Grossman, \& Waters, 2005). Allen and Land (1999) and Thompson (1999) reported that higher levels of attachment security predicted higher levels of sociability, positive social behaviour, popularity and friendships in childhood and adolescence. In contrast, Bosquet and Egeland (2006) found that insecure attachment in infancy predicted negative peer relationships and increased social anxiety in adolescence. Cassidy and Berlin (1994) reported that insecure ambivalently attached children, because they are focused on maintaining and gaining proximity to the primary caregivers, are not prepared to organize relationships that are outside of the parent-child relationship, and often show less adaptive exploratory behaviours in situations that are peer-related. Also as Sroufe, Egeland, Carlson, and Collins (2005, p. 137) found in their longitudinal study, 'situations of novelty, high stimulation, object mastery, and cognitive challenges are especially difficult for those with resistant [ambivalent attachment] histories.'

Vertue (2003) suggested that negative models of the self and others may trigger anxiety sufferers to believe that they are under the constant threat of exclusion or rejection. As a result, they may initiate withdrawal behaviours or clumsy attempts to interact socially, which are likely to be met with negative responses from others confirming their earlier negative suspicions. There is strong support for the idea that peer rejection can heighten social withdrawal (Sroufe et al., 2005). Additionally, peer rejection could be seen as more traumatic in a collectivist society such as Japan's than in an individualistic one, due to the increased importance of group belonging (Hofstede, 1991; Triandis, 1995).

Peer rejection or bullying may be correlated with hikikomori (Borovoy, 2008; Furlong, 2008). In Hattori's (2003) clinical sample of hikikomori, as many as $54 \%$ could recall being rejected by their peers in a cruel form of rejection called ijime (bullying, verbal abuse, physical abuse and obvious shunning (Tanaka, 2001)). While such an experience would undoubtedly be painful for anyone, children and adolescents who are anxiously attached and do not have a secure base of attachment at home may become targets for ijime and in a collectivist society that values peer group belonging, become socially withdrawn, refuse to attend school or consider taking their own lives (Hattori, 2005; Tanaka, 2001; Teo, 2010).

\section{Psychosocial developmental model of hikikomori}

Figure 2 illustrates the psychosocial developmental model of hikikomori emphasizing the influence of attachment, dispositional traits and peer relationships.

This model brings together the earlier research that we have presented thus far (later supported by the data this study collected (see Figure 3)) and organizes it within the context of three developmental stages. In the Early Childhood stage, we see the importance of dispositional 


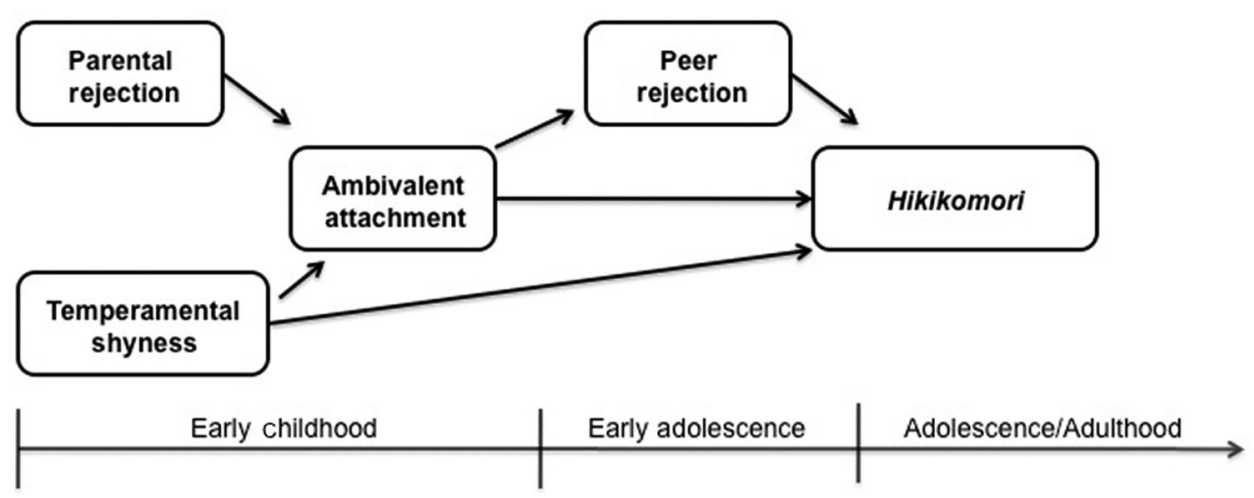

Figure 2. Psychosocial developmental model of hikikomori.

characteristics, maternal behaviour and the security/insecurity of the parent-child attachment, which occur within a cultural context that influences child-rearing approaches.

Upon reaching the Mid-Childhood/Early Adolescence stage, we focus on acceptance by the peer group. While securely attached children with adaptive dispositional features may have little problem transferring their healthy attachment strategies from their parents to their peers, other children who have maladaptive dispositional features and/ or insecure attachment may struggle with peer relations. Many of these children refuse to go to school in late elementary school or middle school (futoko in Japanese), while others continue through the educational system with a growing sense of anxiety.

During this time, as well as in the Late-Adolescence/ Early-Adulthood stage, there is an opportunity for peer-topeer intervention. At times, a peer group may help suffering individuals adjust and become more socially grounded. Other times, however, peers can aggravate these conditions through ijime (bullying) and rejecting behaviours that contribute to social withdrawal.

This model predicts a higher prevalence of ambivalent attachment among hikikomori compared to that of a compaison group. Additionally, recollected instances of maternal rejecting behaviours that would contribute to this attachment style should also be comparatively higher. With regard to dispositional traits, measures of temperament of hikikomori patients should evidence greater sensitivity, reflectivity and discomfort with novelty - especially dealing with social novelty - than would be expected in a comparison group. We also predict the reported experiences of peer rejection should be more frequent and more intense among the hikikomori sample. We propose that the combination of these effects increases the risk of engaging in hikikomori/social withdrawal.

\section{Methods}

\section{Participants}

Hikikomori participants (14 male and 10 female, aged 14-32 $(M=22.84))$ were recruited in Tokyo and Kanagawa from three programmes for hikikomori sufferers: a group therapy centre, a hikikomori day care centre and a church help group. Comorbidity diagnoses included nine with an anxiety disorder, three with a developmental disorder and two with mood disorder. For the remaining 10 people comorbidity was unknown. It is important to note that all of these participants are considered 'former' or 'recovering' hikikomori, either through verbal self-identification $(n=8)$ or through current participation in a treatment group specifically designed for hikikomori. The used definition of hikikomori was the same as listed in the introduction: 'behavior containing both elements of social withdrawal and social isolation'.

A contrast group of 27 males and 32 females, aged 18-24 $(M=20.59)$ were recruited from three universities (two in Tokyo and one in Kanagawa). All participants in both groups reside in the Kanto Region of Japan.

\section{Materials and procedures}

Each participant individually completed culturally appropriate scales measuring trait shyness, attachment, maternal rejections and peer rejection. Table 1 presents the ranges, means and standard deviations for all variables.

Trait Shyness Scale. Created and validated in Japan (Aikawa, 1991), this scale included a sum of five Likert items ( $1=$ 'strongly disagree', 5 = 'strongly agree') measuring the temperamental inhibition experienced in social situations, including making new friends, talking with strangers and being in places with unfamiliar people. These questions included items such as 'it is easy to make new friends', 'I am nervous in places with lots of people' and 'I can speak easily with people I do not know'.

Maternal Attachment Scale. Created and validated in Japan (Honda, 2002), two scales measured insecure/ avoidant attachment and insecure/ambivalent attachment in the mother-child relationship using four-level Likert items ( $1=$ 'disagree', 4 = 'agree'). Insecure/avoidant averaged eight items such as 'my mother doesn't understand me', 'I don't talk to my mother when I am in a difficult situation' and 'I do not want to be close to my mother'. Insecure/ anxious averaged seven items such as 'I am uneasy about whether or not my mother thinks I am a good child', 
Table I. Ranges, means and standard deviations of measures.

\begin{tabular}{|c|c|c|c|c|c|c|c|c|}
\hline \multirow[t]{2}{*}{ Variable } & \multicolumn{4}{|c|}{ Hikikomori group } & \multicolumn{4}{|c|}{ Contrast group } \\
\hline & $n$ & $M$ & SD & Range & $n$ & M & SD & Range \\
\hline Gender & 24 & 1.38 & 0.50 & $\mathrm{I}-2$ & 61 & 1.52 & 0.50 & $\mathrm{I}-2$ \\
\hline Age & 19 & 22.84 & 6.41 & $14-32$ & 61 & 20.59 & 1.23 & $18-24$ \\
\hline Shyness & 24 & 52.83 & 12.27 & $21-70$ & 61 & 46.89 & 9.76 & $18-68$ \\
\hline Avoidance & 24 & 2.21 & 0.70 & $\mathrm{I}-3.50$ & 61 & 2.09 & 0.71 & $|-3.7|$ \\
\hline Ambivalence & 24 & 2.08 & 0.75 & $\mathrm{I}-3.57$ & 61 & 1.51 & 0.51 & $\mathrm{I}-3$ \\
\hline $\begin{array}{l}\text { Adjustment at elementary } \\
\text { school }\end{array}$ & 7 & 3.57 & 1.99 & $\mathrm{I}-7$ & 61 & 3.03 & 1.92 & $\mathrm{I}-7$ \\
\hline Adjustment at middle school & 7 & 5.71 & I.II & $4-7$ & 61 & 3.30 & 1.81 & $\mathrm{I}-7$ \\
\hline Adjustment at high school & 2 & 3.50 & 2.12 & $2-5$ & 61 & 3.46 & 1.71 & $\mathrm{I}-7$ \\
\hline Mushi & 24 & 3.00 & 2.09 & $\mathrm{I}-7$ & 61 & 2.15 & 1.57 & $\mathrm{I}-7$ \\
\hline Threaten & 24 & 3.71 & 1.94 & $\mathrm{I}-7$ & 61 & 2.05 & 1.61 & $\mathrm{I}-7$ \\
\hline Lock-out & 24 & 3.58 & 2.21 & $\mathrm{I}-7$ & 61 & 3.21 & 2.37 & $\mathrm{I}-7$ \\
\hline ljime degree & 24 & 3.85 & 2.31 & $\mathrm{I}-8$ & 61 & 2.41 & 2.15 & $\mathrm{I}-8$ \\
\hline Parental rejection & 24 & 10.29 & 4.44 & $3-18$ & 61 & $7.4 I$ & 4.02 & $3-20$ \\
\hline
\end{tabular}

Note: The variation in sample size is due to response rate.

'I worry about what my mother is thinking' and 'I want my mother to fuss over me more'. High scores indicated insecure attachment.

Recollection of Parental Rejecting Behaviour Scale. This scale, created for this study, contained three seven-level Likert items ( $1=$ 'not at all', $7=$ 'often') measuring recollection of rejecting parental behaviours during childhood: mushi (ignoring), threatening loss of the relationship and locking out of the house.

Peer Rejection (Ijime) Scale. This scale, created for this study, was a single eight-level Likert item $(1=$ 'not experienced', 8 = 'high intensity') measuring the intensity of ijime (bullying) experienced during the school years.

Maladjustment to School Scale. This scale, created for this study, contained three seven-level Likert items $(1=$ 'easy to adjust', $7=$ 'very difficult to adjust') measuring the recollection of the difficulty adjusting to peer group work in elementary, middle and high school. Due to missing data for the high school measure (21 out of 24 in the hikikomori sample), only elementary and middle school are analysed.

Table 2 shows inter-correlations among the variables.

\section{Results}

\section{Greater ambivalent attachment among hikikomori}

To test the hypothesis that there would be a higher prevalence of ambivalent attachment among hikikomori compared to the control group, we performed a 2(group) x 2(gender) analysis of variance (ANOVA). The hikikomori sample scored significantly higher on the Maternal Attachment Scale's measure of ambivalence (means: hikikomori $=2.08$ $(\mathrm{SD}=0.51)$, contrast $=1.52(\mathrm{SD}=0.75) ; F(1,81)=17.57$, $p<.001)$. There was no significant effect of gender. In order to confirm that it is anxious/avoidant attachment that is the best predictor of hikikomori, we performed a regression analysis including scores for both ambivalent and avoidant attachment. As predicted, only ambivalent attachment predicted hikikomori $(b=.40, t(84)=3.93$, $p<.001 ; R 2=.16, F(2,82)=8.06, p<.001)$.

\section{Recollection of more parental rejecting behaviours among hikikomori}

To test the hypothesis that hikikomori participants would recollect more parental rejecting behaviours compared to the contrast group, we performed an ANOVA. The hikikomori sample scored significantly higher on parental rejection (means: hikikomori $=10.29(\mathrm{SD}=4.44)$, contrast $=$ $7.41(\mathrm{SD}=4.02), F(1,83)=8.35, p=.005)$. To determine which of the three types of behaviour were most predictive of hikikomori, we performed a regression analysis, including the parental behaviours, locking out, mushi (ignoring) and threatening loss of relationship. This last factor, threatened loss of relationship, was the only significant predictor of hikikomori $(b=.40, t(84)=3.37, p=.001 ; R 2=.17, F(3$, $81)=5.38, p=.002)$.

\section{Greater trait shyness among hikikomori}

To test the hypothesis that there would be a higher prevalence of trait shyness among hikikomori compared to the control group, we performed a 2(group) x 2(gender) ANOVA. The hikikomori sample scored significantly higher on the Trait Shyness Scale (means: hikikomori $=$ $52.83(\mathrm{SD}=12.27)$, contrast $=46.89(\mathrm{SD}=9.76) ; F(1,81)$ $=4.79, p<.001)$. There was no significant effect of gender. 


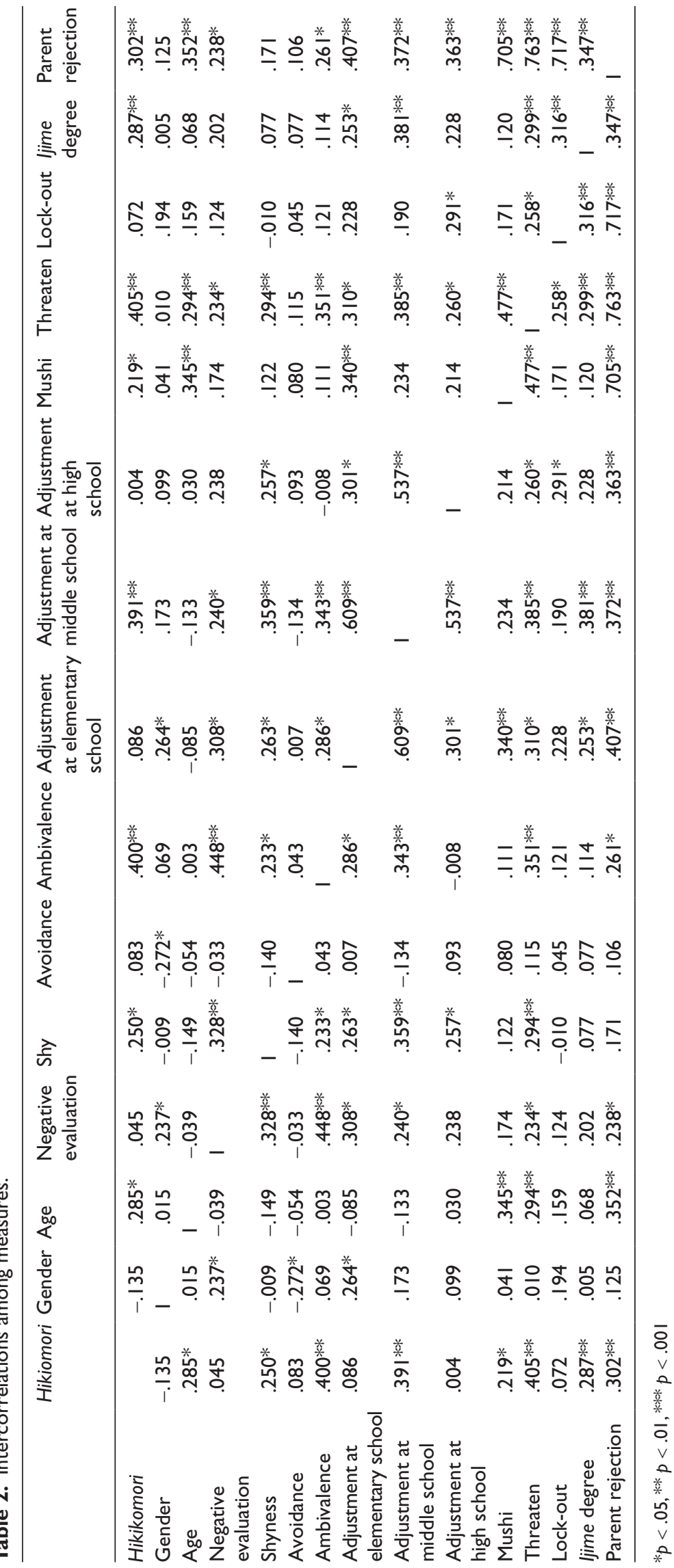




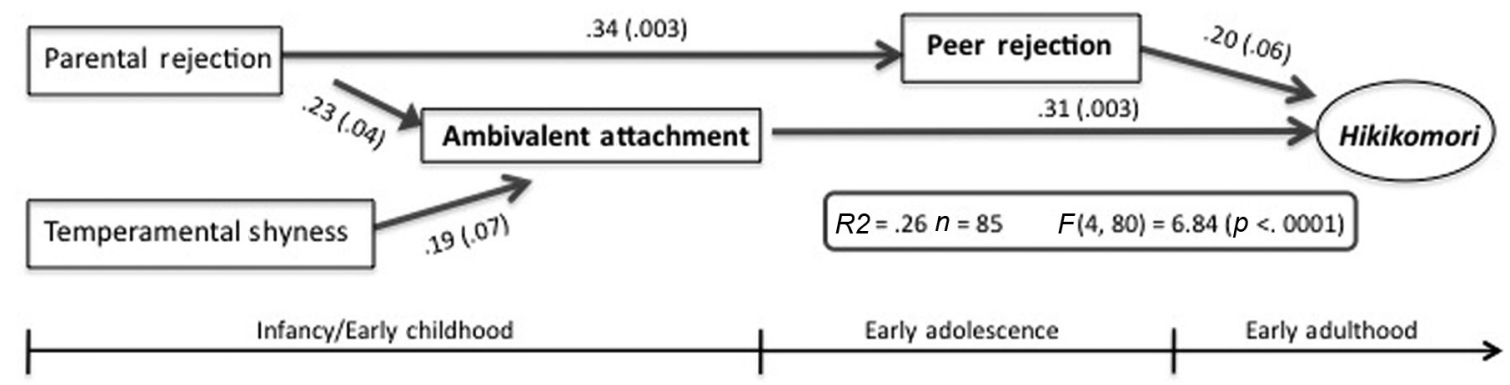

Figure 3. Path analysis of psychosocial developmental model of parental rejection, temperamental shyness, ambivalent attachment and peer rejection predicting hikikomri ( $\beta$ s with significance in parentheses are shown).

\section{Recollection of more peer rejection among hikikomori particularly in early adolescence}

To test the hypothesis that hikikomori participants would recall more peer rejection particularly in early adolescence compared to the contrast group, we performed an ANOVA on recollections of bullying (ijime) in childhood, and adjustment to the peer group in elementary and middle school. The hikikomori participants scored significantly higher on peer rejection (means: hikikomori $=3.85(\mathrm{SD}=2.3)$, contrast $=2.4(\mathrm{SD}=2.15) ; F(1,83)=7.47, p=.008)$. Further, we found support for the developmental significance of early adolescence peer rejection. While the difference between hikikomori and the comparison group in recollections of elementary school peer group adjustment were non-significant $(F<1)$, there was a significantly greater recollection of peer group discomfort among the hikikomori than the contrast group in middle school (means: hikikomori $=5.71(\mathrm{SD}=1.11)$, contrast $=3.3(\mathrm{SD}=1.81)$; $F(1,66)=11.88, p=.001)$.

\section{Path analysis of the psychosocial developmental model of hikikomori}

To test the model's assumptions that early parental rejecting behaviour affects attachment and security, which when coupled with early adolescent peer rejection and shy temperamental disposition combines to predict social withdrawal/hikikomori, we performed a path analysis of these predictors. A path analysis uses successive regression analyses to find the best sequential set of variables to predict an outcome. Each regression analysis shows the strongest predictions of an outcome, and combined they show the best path to predict the dependent variable (Figure 3).

The path analysis revealed that while parental rejection did significantly predict ambivalent attachment and peer rejection, it did not directly predict hikikomori. As the model predicted, it is the combination of ambivalent attachment and peer rejection that together significantly predict hikikomori. Trait shyness showed a trend in impacting ambivalent attachment, but did not directly predict hikikomori.

\section{Discussion}

In this paper, we began by presenting an overview of hikikomori as a condition with negative social implications for Japan. We followed by giving an overview of attachment and its related factors, explaining the connection between social withdrawal and insecure attachment, noting especially the impact of maternal behaviour in the development of internal working models in the child. We outlined some Japanese behaviours with regards to attachment, childrearing and social situations, which have implications for social engagement and social withdrawal. Finally, we mentioned how the Japanese culture of harmony may increase the psychological impact of peer rejection via ijime (bullying) as a stressor that could contribute to hikikomori.

Our psychosocial developmental model of hikikomori suggests that some children (arguably the majority) are better equipped to make the developmental transitions to adulthood, possessing either secure parental attachment, adaptive dispositional features or both. However, others are less equipped and less ready to make these transitions, and may follow a pattern of dispositional sensitivity, parental rejection and anxious-insecure attachment, followed by peer rejection.

Our results show that parental rejection and temperament individually affected hikikomori via their influence on attachment and peer rejection. Attachment theory implies that lifelong relationships and effective social functioning are based on the first relationship model of the infantparent attachment. This early internal working model in which a sense of efficacy as well as a sense of trust in self and others frees up the infant, child, adolescent and adult to explore, to take risks, to engage socially, especially during the vulnerable transitions from childhood to adolescence when peers replace parents as the focus of social relations. If that internal working model suggests lack of trust in self and others, then rejection by peers is more likely to happen, which increases the risk of social withdrawal, and in the extreme, isolation or hikikomori. Parental rejection early on (as recollected by our hikikomori sample) predicted peer bullying and rejection in early adolescence as if the internal working model of the 'other' (the bullying parent) carries 
over to the relationship model of the self, who is also rejected by bullying peers. In our psychosocial developmental model, risk for social withdrawal/hikikomori is increased through the combination of anxious attachment and peer rejection.

Temperamental shyness by itself when coupled with anxious attachment and peer rejection does not directly predict hikikomori. Booth-LaForce and Oxford's (2008) longitudinal research on social withdrawal in American children differentiates between aspects of temperament that predict social withdrawal in middle childhood and those that do not. They found that being shy did not predict social withdrawal, but dysregulated temperament, a tendency to act out impulsively, predicted both later experiences of bullying and social withdrawal. Further studies of temperament among hikikomori are needed to determine whether other aspects of temperament may contribute to social withdrawal more directly.

\section{Implications for research and practice}

Our model implies that treatment and prevention may require attention to attachment insecurities in early childhood, and to peer rejection in middle childhood and/or early adolescence. We believe it is helpful in understanding hikikomori to first understand how the attachment system balances security with exploration and the anxiety associated with novelty and challenge (Bowlby, 1982). As we examined hikikomori we saw that the manifestations of this disorder in adolescents and young adults mimic the behaviour of anxiously attached children. Both anxiously attached children and hikikomori sufferers seem less able to tolerate anxiety in new situations and seem unable to rely on an internalized sense of security - the internal working model that allows mediation between anxiety and exploration (Granqvist \& Dickie, 2006).

We suggest that at the developmental moment when the attachment system shifts from reliance on caregivers to reliance on peers (Friedlmeier \& Granqvist, 2006), insecurely attached children are especially challenged by the anxiety of new expectations with the social system. These children might be helped through this anxiety if they have access to a sensitively tuned-in peer group that facilitates the transition. If, however, these at-risk adolescents are exposed instead to rejection or, at the extreme, bullying, then we argue that they are unable to overcome the heightened anxiety they experienced and seek the relative safety of home where they defensively retreat.

Although successful treatment of some behaviour disorders does not necessarily require understanding of the etiology of the disorder, we believe that in general, interventions and treatments of hikikomori could be improved by using a psychosocial developmental model. As our model suggests, if in early childhood children experience ambivalent attachment, they may be more susceptible to negative peer reactions. When this occurs, the developmental transfer of safe haven functions from caregivers to peers is disrupted (Bosquet \& Egeland, 2006).

Using our model, intervention and treatment could occur at any one of the developmental periods. Schools may need projects that incorporate at-risk children into peer groups and identify situations in which peer rejection or bullying is occurring so that they can intervene quickly. Of course awareness and intervention in bullying or peer rejection are currently being explored in Western cultures as well (Powell \& Ladd, 2010; Reijntjes, Kamthuis, Prinzie, \& Telch, 2010). Peer rejection is painful for children in any culture, but we suggest its effects may be more devastating in children who come from more collectivist societies, where expectations of peer cooperation, acceptance and harmony prevail.

Treatment of hikikomori focusing on the attachment aspect of the model could incorporate the concept of a substitute attachment figure, which could provide the client with both a secure base and revised internal working model. The idea of a substitute attachment figure has been explored most thoroughly in research on the ways in which children and adults use God as the source of a secure base and safe haven (for a review, see Granqvist \& Dickie, 2006). However, the therapist-client relationship certainly contains elements of the attachment system, including trust, continuity and a safe base to explore self and others. Building a new efficacious internal working model of self and others will depend primarily on the experience of a secure and trusting relationship. Cognitive techniques could help hikikomori patients more accurately read social cues in a given context and interpret their anxiety as a reflection of the situation rather than their own deficiencies. Direct social skill training would facilitate feelings of efficacy and provide something for hikikomori patients to do when different social skills are required. Finally, the model suggests that once a 'secure base', trusting relationship is established, the therapist could incorporate group therapy that allows for the developmental transition of 'safe haven' attachment functions from a primary attachment to peers.

\section{Limitations}

Further research on this model is needed. There were some limitations of this study. First, we used retrospective measures of parental and peer behaviour as well as school adjustment. This presents a risk for recall bias and longitudinal studies that measure attachment in childhood and peer rejection in early adolescence would strengthen these results. Also, the sample size of the hikikomori group was relatively small $(N=24)$ and needs to be replicated. If a replicated study manages to secure a larger sample size it may be profitable to separate the hikikomori group into separate groups of psychiatric disorders (if presenting or known) to better understand the diversity of this group and 
how it relates to the parental attachment. Also, if a group of 'pure' (not in treatment or recovering) hikikomori were able to be sampled, we would be able to see if there is a difference between the attachment patterns between 'pure' and 'recovering' hikikomori. Nonetheless, as one of the first studies to empirically explore the relationship of hikikomori with parental and peer relationships, we believe that it contributes to understanding this growing phenomenon. Research on treatment efficacy of therapies that employ an explicit programme of 'attachment' development between the therapist and client and programmes that develop 'practice peer relationships' are needed to determine whether this model is helpful in the treatment of hikikomori. Furthermore, this model, while pulling together much information from biological and sociological resources, mainly addresses the psychosocial development of the individual, and therefore does not explain what is happening on the societal level or how the country's economic situation or educational system contribute to this condition. While we believe that this model could be very helpful in understanding hikikomori, it may be best used in conjunction with a greater sociological understanding of the surrounding social system and its impact on these individuals.

\section{Conclusion}

Hikikomori in Japan and social withdrawal among adolescents and young adults around the world represent a terrible loss not only to families and individuals struggling with these disorders but to society as a whole. While we cannot account for the whole of the problem in our model, we hope that this will help pave the way for the needed prevention, intervention and treatment. With an empirically based theoretical model to point the way, we may begin to make progress.

\section{Acknowledgements}

We thank Professor Fumihito 'Andy' Nakajima (Department of Modern and Classical Languages, Hope College) for his inspiration and support. His comments and cultural insight were invaluable as this theory was being outlined. Also, we would like to thank Hikaru Kondo (Meiji Gakuin University, Japan) for her translation of Japanese journal articles and Patricia Roehling and John Shaughnessy for their careful reading of this article. Finally, we would like to thank the participants for helping us understand their experiences.

\section{References}

Aikawa, A. (1991). Making the characteristic shyness scale with the consideration of reliability and validity research. Psychological Research, 62, 149-155.

Ainsworth, M. D. S., Blehar, M. C., Waters, E., \& Wall, S. (1978). Patterns of attachment: A psychological study of the strange situation. Oxford, UK: Lawrence Erlbaum.

Allen, J. P. \& Land, D. (1999). Attachment in adolescence. In J. Cassidy \& P. R. Shaver (Eds), Handbook of attachment:
Theory, research, and clinical applications (pp. 319-335). New York: Guilford Press.

American Psychiatric Association. (2000). Diagnostic and statistical manual of mental disorders, 4th edn, text revision. Washington, DC: American Psychiatric Association.

Aoki, S. (2010). Government estimates 700,000 hikikomori and 1.5 million at risk. Yomiuri Shinbun, 24 July.

Aron, E. N. (1999). High sensitivity as one source of fearfulness and shyness. In J. Schulkin \& L. A. Schmidt (Eds), Extreme fear, shyness and social phobia (pp. 251-272). New York: Oxford University Press.

Asahi Shinbun. (2000). The scary 'real world' of reclusive youngsters, 5 October. Retrieved from http://dir.groups.yahoo.com/ group/LearningCommunities/message/946?unwrap=1\&var=1

Booth-LaForce, C. \& Oxford, M. L. (2008). Trajectories of social withdrawal from grades 1 to 6: Prediction from early parenting, attachment, and temperament. Developmental Psychology, 44, $1298-1313$.

Borovoy, A. (2008). Japan's hidden youths: Mainstreaming the emotionally distressed in Japan. Culture, Medicine and Psychiatry, 32, 552-576.

Bosquet, M. \& Egeland, B. (2006). The development and maintenance of anxiety symptoms from infancy through adolescence in a longitudinal sample. Development and Psychopathology, $18,517-550$.

Bowlby, J. (1969). Disruption of affectional bonds and its effects on behavior. Canada's Mental Health Supplement, $59,12-33$.

Bowlby, J. (1973). Attachment and loss: Vol. 2. Separation anxiety and anger. New York: Basic Books.

Bowlby, J. (1982). Attachment and loss: Vol. 1. Attachment. London, UK: Tavistock (originally published 1969).

Cassidy, J. (1994). Emotion regulation: Influences of attachment relationships. Monographs of the Society for Research in Child Development, 59, 228-250.

Cassidy, J. \& Berlin, L. (1994). The insecure/ambivalent pattern of attachment: Theory and research. Child Development, 65, 971-991.

Cassidy, J. \& Shaver, P. (1999). Handbook of attachment. New York: The Guilford Press.

Caudill, W. \& Weinstein, H. (1969). Maternal care and infant behavior in Japan and America. Psychiatry, 32, 12-43.

Chen, S. \& Miyake, K. (1986). Japanese studies of infant development. In H. Stevenson, H. Azuma, \& K. Hakuta (Eds), Child development and education in Japan (pp. 135-146). New York: W. H. Freeman \& Company.

Cunha, M., Soares, I., \& Pinto-Gouveia, J. (2008). The role of individual temperament, family and peers in social anxiety disorder: A controlled study. International Journal of Clinical and Health Psychology, 8, 631-655.

Fraley, R. C. \& Davis, K. E. (1997). Attachment formation and transfer in young adults' close friendships and romantic relationships. Personal Relationships, 4, 131-144.

Friedlmeier, W. \& Granqvist, P. (2006). Attachment transfer among German and Swedish adolescents: A prospective longitudinal study. Personal Relationships, 13, 261-279.

Furlong, A. (2008). The Japanese hikikomori phenomenon: Acute social withdrawal among young people. Sociological Review, 56, 309-325. 
Garcia-Campayo, J., Alda, M., Sobradiel, N., \& Sanz, A. B. (2007). A case report of hikikomori in Spain. Medicina Clinica, 129, 318-319.

Genuis, M. (1994). Long-term consequences of childhood attachment: Implications for counselling adolescents. International Journal for the Advancement of Counselling, 17, 263-274.

Goldsmith, H. H. \& Alansky, J. A. (1987). Maternal and infant temperamental predictors of attachment: A meta-analytic review. Journal of Consulting and Clinical Psychology, 55, 805-816.

Granqvist, P. \& Dickie, J. (2006). Attachment and spiritual development in childhood and adolescence. In E. Roehlkepartain, P. King, L. Wagener, \& P. Benson (Eds), The handbook of spiritual development in childhood and adolescence (pp. 197210). Thousand Oaks, CA: Sage.

Grossman, K., Grossman, K., \& Waters, E. (2005). Attachment from infancy to adulthood: The major longitudinal studies, $1 \mathrm{st}$ edn. New York: The Guilford Press.

Hastings, P. D., Nuselovici, J. N., Rubin, K. H., \& Cheah, C. S. L. (2010). Shyness, parenting, and parent-child relationships. In K. H. Rubin \& R. J. Coplan (Eds), The development of shyness and social withdrawal (pp. 107-130). New York: The Guilford Press.

Hattori, Y. (2003). Keynote panel: Social withdrawal/DDNOS through faulty attachment between parents and child in seemingly well-functioning Japanese families. International Conference on Trauma, Attachment, and Dissociation: Transforming Trauma, The Delphi Centre, Melbourne, Australia, 14 September.

Hattori, Y. (2005). Social withdrawal in Japanese youth: A case study of 35 hikikomori clients. Journal of Practice Trauma, 4, 181-201.

Hazan, C. \& Zeifman, D. (1999). Pair bonds as attachments: Evaluating the evidence. In J. Cassidy \& P. R. Shaver (Eds), Handbook of attachment theory and research (pp. 336-355). New York: The Guilford Press.

Hofstede, G. (1991). Cultures and organizations: Software of the mind. London, UK: McGraw-Hill.

Honda, J. (2002). Making the 'the attachment with the mother' the measurement standard of a child. Counselling Research, $35(1), 245-246$.

Isabella, R. A. (1993). Origins of attachment: Maternal interactive behavior across the first year. Child Development, 64, 605-621.

Kawanishi, Y. (2006). Families coping with mental illness: Stories from the US and Japan. New York: Routledge.

Koshiba, Y. (2007). A study of family functioning in hikikomori (social withdrawal). Hiroshima University's Study of Health Preservation Journal (Koudaihokengaku), 6, 95-101.

Koyama, A., Miyake, Y., Kawakami, N., Tsuchiya, M., \& Tachimori, H. (2010). Lifetime prevalence, psychiatric comorbidity and demographic correlates of 'hikikomori' in a community population in Japan. Psychiatry Research, 176(1), 69-74.

Lamb, M. E., Thompson, R. A., Gardner, W., \& Charnov, E. L. (1985). Infant-mother attachment: The origins and developmental significance of individual differences in strange situation behavior. Hillsdale, NJ: Lawrence Erlbaum Associates.

Larimer, T. (2001). Japan's wild ones. Time, 8 January.

Lebra, T. S. (1976). Japanese patterns of behavior. Honolulu, HI: University Press of Hawaii.
Maccoby, E. E. \& Jacklin, C. N. (1987). Gender segregation in childhood. In H. W. Reese (Ed.), Advances in child development and behavior (pp. 239-288). New York: Academic Press.

Main, M. \& Hesse, E. (1990). Parents' unresolved traumatic experiences are related to infant disorganization status. In M. T. Greenberg, D. Cicchetti, \& E. M. Cummings (Eds), Attachment in the preschool years: Theory, research, and intervention (pp. 161-182). Chicago, IL: University of Chicago Press.

Ministry of Health, Labour and Welfare. (2003). Community mental health intervention guidelines aimed at socially withdrawn teenagers and young adults. Tokyo, Japan: Ministry of Health, Labour and Welfare.

Miyake, K., Campos, J. J., Kagan, J., \& Bradshaw, D. L. (1986). Issues in socioemotional development. In H. Stevenson, H. Azurna, \& K. Hakuta (Eds), Child development and education in Japan (pp. 239-261). New York: Freeman.

Miyake, K., Chen, S., \& Campos, J. (1985). Infant temperament, mother's mode of interaction, and attachment in Japan: An interim report. Monographs of the Society for Research in Child Development, 50, 276-297.

Miyake, Y. (2002). A survey of hikikomori based on an epidemiological study. Tokyo, Japan: Health and Labour Sciences Research Grants Board.

Nakajima, T., Tsukamoto, C., Ooshige, K., Kishi, Y., \& Oota, J. (2008). Seishinka kyuuseiki iryou ni okeru hikikomori seinen no jittai to seishin igakuteki chiryou ni kan suru kenkyuu [Research on the characteristics and psychiatric treatment of youth with acute social withdrawal]. Kokoro no Kenkou Kagaku Kenkyuu, 19-24.

Plomin, R. \& Bergeman, C. S. (1991). The nature of nurture: Genetic influence on 'environmental' measures. Behavioral and Brain Sciences, 14, 373-427.

Powell, M. D. \& Ladd, L. D. (2010). Bullying: A review of the literature and implications for family therapists. American Journal of Family Therapy, 38, 189-206

Rees, P. (2002). Hikikomori violence. BBC News World Edition, 18 October. Retrieved from http://news.bbc.co.uk/2/hi/ programmes/correspondent/2336883.stm

Reijntjes, A., Kamphuis, J. H., Prinzie, P., \& Telch, M. J. (2010). Peer victimization and internalizing problems in children: A meta-analysis of longitudinal studies. Child Abuse \& Neglect, 34, 244-252.

Rothbaum, F., Pott, M., Azuma, H., Miyake, K., \& Weisz, J. (2000). The development of close relationships in Japan and the United States: Paths of symbiotic harmony and generative tension. Child Development, 71, 1121-1142.

Rothbaum, F., Weisz, J., Pott, M., Miyake, K., \& Morelli, G. (2000). Attachment and culture: Security in the United States and Japan. American Psychologist, 55, 1093-1104.

Rubin, K. H., Chen, X., McDougall, P., Bowker, A., \& McKinnon, J. (1995). The Waterloo Longitudinal Project: Predicting internalizing and externalizing problems in adolescence. Development \& Psychopathology, 7, 751-764.

Rubin, K. H., Hastings, P. D., Stewart, S. L., Henderson, H. A., \& Chen, X. (1997). The consistency and concomitants of inhibition: Some of the children, all of the time. Child Development, 68, 467-483. 
Saito, T. (1998). Shakaiteki hikikomori [Social withdrawal]. Tokyo, Japan: PHP Kenkyuujo.

Saito, T. (2003). Individual treatment and therapy for Hikikomori cases. Seishen Igaku [Clinical Psychiatry], 45, 263-269.

Sakamoto, N., Martin, R. G., Kumano, H., Kuboki, T., \& Al Adawi, S. (2005). Hikikomori, is it a culture-reactive or culture-bound syndrome? Nidotherapy and a clinical vignette from Oman. International Journal of Psychiatry in Medicine, 35, 191-198.

Scher, C. D. (2000). Mechanisms of the relationship between childhood attachment and adult anxiety and depression. Dissertation Abstracts International: Section B: The Sciences and Engineering, 61(5-B), 2781.

Sroufe, L. A., Egeland, B., Carlson, E., \& Collins, W. A. (2005). The development of the person: The Minnesota study of risk and adaptation from birth to adulthood. New York: The Guilford Press.

Suwa, M. \& Suzuki, K. (2002). Psychopathological features of 'primary social withdrawal'. Seishin Shinkeigaku Zasshi, 104, $1228-1241$.

Takahashi, Y. (1990). Separation distress of Japanese infants in the strange situation. Research and Clinical Center for Child Development, 12, 141-150.

Tanaka, T. (2001). The identity formation of the victim of shunning. School Psychology International, 22, 463-476.

Teo, A. R. (2010). A new form of social withdrawal in Japan: A review of hikikomori. International Journal of Social Psychiatry, 56, 178-185.

Teo, A. R. \& Gaw, A.C. (2010). Hikikomori, a Japanese culture culture-bound syndrome of social withdrawal? A proposal for DSM-5. Journal of Nervous and Mental Disease, 198, 444-449.

Thomas, A. \& Chess, S. (1977). Temperament and development. Oxford, UK: Brunner/Mazel.

Thompson, R. A. (1999). Early attachment and later development. In J. Cassidy \& P. R. Shaver (Eds), Handbook of attachment:
Theory, research, and clinical applications (pp. 265-286). New York: The Guilford Press.

Triandis, H. (1995). Individualism and collectivism. Boulder, CO: Westview.

Tsujimoto, T., Daimon, K., Izumi, K., Sawai, M., \& Iwashige, T. (2007). School-refusal and social-withdrawal in the clinical setting at a psychiatric medical. Seishin Shinkeigaku Zasshi, 109, 313-320. [in Japanese]

Ujiie, T. \& Miyake, K. (1984). Responses to the strange situation in Japanese infants. Research \& Clinical Center for Child Development, Annual Report 1983-1984, 27-36.

Van IJzendoorn, M. H. \& Kroonenberg, P. M. (1988). Crosscultural patterns of attachment: A meta-analysis of the strange situation. Child Development, 59, 147-156.

Vertue, F. M. (2003). From adaptive emotion to dysfunction: An attachment perspective on social anxiety disorder. Personality and Social Psychology Review, 7, 170-191.

Vogel, S. H. \& Vogel, E. F. (1961). Family security, personal immaturity, and emotional health in a Japanese sample. Marriage and Family Planning, 23, 161-166.

Weinfield, N. S., Sroufe, L. A., Egeland, B., \& Carlson, E. A. (1999). The nature of individual differences in infant-caregiver attachment. In J. Cassidy \& P. R. Shaver (Eds), Handbook of attachment: Theory, research, and clinical applications (pp. 68-88). New York: The Guilford Press.

Wong, V. (2009). Youth locked in time and space? Defining features of social withdrawal and practice implications. Journal of Social Work Practice, 23, 337-352.

Yukawa, S., Tokuda, H., \& Sato, J. (2007). Attachment style, self-concealment, and interpersonal distance among Japanese undergraduates. Perceptual and Motor Skills, 104, 1255-1261.

Zielenziger, M. (2006). Shutting out the sun: How Japan created its own lost generation. New York: Random House. 